\title{
Non-abelian representations of the slim dense near hexagons on 81 and 243 points
}

\author{
B. De Bruyn • B.K. Sahoo • N.S.N. Sastry
}

Received: 13 November 2009 / Accepted: 17 May 2010 / Published online: 3 June 2010

(C) Springer Science+Business Media, LLC 2010

\begin{abstract}
We prove that the near hexagon $Q(5,2) \times \mathbb{L}_{3}$ has a non-abelian representation in the extra-special 2-group $2_{+}^{1+12}$ and that the near hexagon $Q(5,2) \otimes Q(5,2)$ has a non-abelian representation in the extra-special 2-group $2_{-}^{1+18}$. The description of the non-abelian representation of $Q(5,2) \otimes Q(5,2)$ makes use of a new combinatorial construction of this near hexagon.
\end{abstract}

Keywords Near hexagon · Non-abelian representation · Extra-special 2-group

\section{Introduction}

Let $\mathcal{S}=(P, L)$ be a partial linear space with point set $P$ and line set $L$. We suppose that $\mathcal{S}$ is $s l i m$, i.e., that every line of $\mathcal{S}$ is incident with precisely three points. For distinct points $x, y \in P$, we write $x \sim y$ if they are collinear. In that case, we denote by $x y$ the unique line containing $x$ and $y$ and define $x * y$ by $x y=\{x, y, x * y\}$. For $x \in P$, we define $x^{\perp}:=\{x\} \cup\{y \in P: y \sim x\}$. If $x, y \in P$, then $d(x, y)$ denotes the distance between $x$ and $y$ in the collinearity graph of $\mathcal{S}$.

B. De Bruyn (凶)

Department of Mathematics, Ghent University, Krijglaan 281 (S22), 9000 Gent, Belgium e-mail: bdb@cage.ugent.be

B.K. Sahoo

School of Mathematical Sciences, National Institute of Science Education and Research, Sainik School Post, Bhubaneswar 751005, Orissa, India e-mail: bksahoo@niser.ac.in

N.S.N. Sastry

Statistics and Mathematics Unit, Indian Statistical Institute, Mysore Road, R. V. College Post, Bangalore 560059, India

e-mail: nsastry@isibang.ac.in 
A representation $[9, \mathrm{p} .525]$ of $\mathcal{S}$ is a pair $(R, \psi)$, where $R$ is a group and $\psi$ is a mapping from $P$ to the set of involutions of $R$, satisfying:

(R1) $R$ is generated by the image of $\psi$.

(R2) $\psi$ is one-one on each line $\{x, y, x * y\}$ of $\mathcal{S}$ and $\psi(x) \psi(y)=\psi(x * y)$.

Notice that if $x \sim y$, then $\psi(x)$ and $\psi(y)$ necessarily commute by Condition (R2). The group $R$ is called a representation group of $\mathcal{S}$. A representation $(R, \psi)$ of $\mathcal{S}$ is faithful if $\psi$ is injective and is abelian or non-abelian according as $R$ is abelian or not. Note that, in [9], 'non-abelian representation' means that 'the representation group is not necessarily abelian'. Abelian representations are called embeddings in the literature. For an abelian representation, the representation group is an elementary abelian 2-group and hence can be considered as a vector space over the field $\mathbb{F}_{2}$ with two elements. We refer to [8] and [12, Sects. 1 and 2] for more on representations of partial linear spaces with $p+1$ points per line, where $p$ is a prime.

A finite 2-group $G$ is called extra-special if its Frattini subgroup $\Phi(G)$, its commutator subgroup $G^{\prime}=[G, G]$ and its center $Z(G)$ coincide and have order 2 . We refer to [5, Sect. 20, pp. 78-79] or [6, Chap. 5, Sect. 5] for the properties of extraspecial 2-groups which we will mention now. An extra-special 2-group is of order $2^{1+2 m}$ for some integer $m \geq 1$. Let $D_{8}$ and $Q_{8}$, respectively, denote the dihedral and the quaternion groups of order 8 . A non-abelian 2-group of order 8 is extra-special and is isomorphic to either $D_{8}$ or $Q_{8}$. If $G$ is an extra-special 2-group of order $2^{1+2 m}$, $m \geq 1$, then the exponent of $G$ is 4 and either $G$ is a central product of $m$ copies of $D_{8}$, or $G$ is a central product of $m-1$ copies of $D_{8}$ and one copy of $Q_{8}$. If the former (respectively, latter) case occurs, then the extra-special 2-group is denoted by $2_{+}^{1+2 m}$ (respectively, $2_{-}^{1+2 m}$ ).

A partial linear space $\mathcal{S}=(P, L)$ is called a near polygon if for every point $p$ and every line $L$, there exists a unique point on $L$ nearest to $p$. If $d$ is the maximal distance between two points of $\mathcal{S}$, then the near polygon is also called a near $2 d$-gon. A near polygon is called dense if every line is incident with at least three points and if every two points at distance 2 have at least two common neighbors. By [1], there are (up to isomorphism) $11 \mathrm{slim}$ dense near hexagons. The paper [13] initiated the study of the non-abelian representations of these dense near hexagons.

Suppose $(R, \psi)$ is a non-abelian representation of a slim dense near hexagon. Then by [13, Proposition 4.1, p. 205], $(R, \psi)$ is necessarily faithful and for $x, y \in P$, $[\psi(x), \psi(y)] \neq 1$ if and only if $x$ and $y$ are at maximal distance 3 from each other. If $\mathcal{S}$ is the (up to isomorphism) unique slim dense near hexagon on 81 points, which will be denoted by $Q(5,2) \times \mathbb{L}_{3}$ in the sequel, then it was shown in [13, Theorem 1.6, p. 199] that $R$ is necessarily isomorphic to the extra-special 2-group $2_{+}^{1+12}$. If $\mathcal{S}$ is the (up to isomorphism) unique slim dense near hexagon on 243 points, which will be denoted by $Q(5,2) \otimes Q(5,2)$ in the sequel, then it was shown in [13, Theorem 1.6, p. 199], that $R$ is necessarily isomorphic to the extra-special 2 -group $2_{-}^{1+18}$. The question whether such non-abelian representations exist remained, however, unanswered in [13]. The following theorem, which is the main result of this paper, deals with these existence problems. 


\section{Theorem 1.1}

(1) The slim dense near hexagon $Q(5,2) \times \mathbb{L}_{3}$ has a non-abelian representation in the extra-special 2-group $2_{+}^{1+12}$.

(2) The slim dense near hexagon $Q(5,2) \otimes Q(5,2)$ has a non-abelian representation in the extra-special 2-group $2_{-}^{1+18}$.

The slim dense near hexagon $Q(5,2) \otimes Q(5,2)$ has many substructures isomorphic to $Q(5,2) \times \mathbb{L}_{3}$. We will describe a non-abelian representation of $Q(5,2) \times \mathbb{L}_{3}$ in Sect. 4. In Sect. 5, we will use this to construct a non-abelian representation of $Q(5,2) \otimes Q(5,2)$. To describe the non-abelian representation of $Q(5,2) \otimes Q(5,2)$, we make use of a model of $Q(5,2) \otimes Q(5,2)$ which we discuss in Sects. 2 and 3.

Remark Two other constructions of non-abelian representations of slim dense near polygons, in particular, of the slim dense near hexagons on 105 and 135 points, can be found in [10].

\section{The point-line geometry $\mathcal{S}_{\theta}$}

Near quadrangles are usually called generalized quadrangles (GQs). A GQ is said to be of $\operatorname{order}(s, t)$ if every line is incident with precisely $s+1$ points and if every point is incident with precisely $t+1$ lines. Up to isomorphism, there exist unique GQs of order $(2,2)$ and $(2,4)$, see, e.g., [11]. These GQs are denoted by $W(2)$ and $Q(5,2)$, respectively. A spread of a point-line geometry is a set of lines partitioning its point set. A spread $S$ of $Q(5,2)$ is called a spread of symmetry if for every line $L \in S$ and every two points $x_{1}, x_{2} \in L$, there exists an automorphism of $Q(5,2)$ fixing each line of $S$ and mapping $x_{1}$ to $x_{2}$. By [2, Sect. 7.1], $Q(5,2)$ has (up to isomorphism) a unique spread of symmetry.

Now, suppose $S$ is a given spread of symmetry of $Q(5,2)$. If $L_{1}$ and $L_{2}$ are two distinct lines of $S$ and if $G$ denotes the unique $(3 \times 3)$-subgrid of $Q(5,2)$ containing $L_{1}$ and $L_{2}$, then the unique line $L_{3}$ of $G$ disjoint from $L_{1}$ and $L_{2}$ is also contained in $S$.

Suppose $\theta$ is a map from $S \times S$ to $\mathbb{Z}_{3}$ (the additive group of order three) satisfying the following property:

(*) If $L_{1}, L_{2}, L_{3}$ are three lines of $S$ contained in a grid of $Q(5,2)$, then $\theta\left(L_{1}, L_{2}\right)+$ $\theta\left(L_{2}, L_{3}\right)=\theta\left(L_{1}, L_{3}\right)$.

Notice that $\theta(L, L)=0$ and $\theta(M, L)=-\theta(L, M)$ for all $L, M \in S$. With $\theta$, there is associated a point-line geometry $\mathcal{S}_{\theta}$. The points of $\mathcal{S}_{\theta}$ are of four types:

$(P 1)$ The points $x$ of $Q(5,2)$.

$(P 2)$ The symbols $\bar{x}$, where $x$ is a point of $Q(5,2)$.

(P3) The symbols $\overline{\bar{x}}$, where $x$ is a point of $Q(5,2)$.

(P4) The triples $(x, y, i)$, where $i \in \mathbb{Z}_{3}$ and $x, y$ are distinct collinear points of $Q(5,2)$ satisfying $x y \in S$.

The lines of $\mathcal{S}_{\theta}$ are of nine types: 
(L1) The lines $\{x, y, z\}$ of $Q(5,2)$.

(L2) The sets $\{\bar{x}, \bar{y}, \bar{z}\}$, where $\{x, y, z\}$ is a line of $Q(5,2)$.

(L3) The sets $\{\overline{\bar{x}}, \overline{\bar{y}}, \overline{\bar{z}}\}$, where $\{x, y, z\}$ is a line of $Q(5,2)$.

(L4) The sets $\{x, \bar{x}, \overline{\bar{x}}\}$, where $x$ is a point of $Q(5,2)$.

(L5) The sets $\{a,(a, b, i),(a, c, i)\}$, where $i \in \mathbb{Z}_{3}$ and $\{a, b, c\} \in S$.

(L6) The sets $\{\bar{a},(b, a, i),(c, a, i)\}$, where $i \in \mathbb{Z}_{3}$ and $\{a, b, c\} \in S$.

(L7) The sets $\{\overline{\bar{a}},(b, c, i),(c, b, i)\}$, where $i \in \mathbb{Z}_{3}$ and $\{a, b, c\} \in S$.

(L8) The sets $\{(a, b, i),(b, c, j),(c, a, k)\}$, where $\{i, j, k\}=\mathbb{Z}_{3}$ and $\{a, b, c\}$ is a line belonging to $S$.

(L9) The sets $\{(a, u, i),(b, v, j),(c, w, k)\}$, where (i) $\{a, b, c\}$ and $\{u, v, w\}$ are two disjoint lines of $Q(5,2)$; (ii) $d(a, u)=d(b, v)=d(c, w)=1$; (iii) $a u, b v, c w \in$ $S$; (iv) $j=i+\theta(a u, b v), k=i+\theta(a u, c w)$.

Incidence is containment. One can easily show that $\mathcal{S}_{\theta}$ is a partial linear space. In order to show that two distinct points of $\mathcal{S}_{\theta}$ are contained in at most one line of Type (L9), one has to make use of Property (*).

\section{An isomorphism $Q(5,2) \otimes Q(5,2) \cong \mathcal{S}_{\theta}$}

The aim of this section is to show that the slim dense near hexagon $Q(5,2) \otimes Q(5,2)$ is isomorphic to a point-line geometry $\mathcal{S}_{\theta}$ for a suitable spread of symmetry $S$ of $Q(5,2)$ and a suitable map $\theta: S \times S \rightarrow \mathbb{Z}_{3}$ satisfying Property (*). We start with recalling some known properties of the near hexagon $Q(5,2) \otimes Q(5,2)$.

(1) Every two points $x$ and $y$ of $Q(5,2) \otimes Q(5,2)$ are contained in a unique convex subspace of diameter 2 , called a quad. The points and lines which are contained in a given quad define a GQ which is isomorphic to either the $(3 \times 3)$-grid or $Q(5,2)$.

(2) If $Q$ is a $Q(5,2)$-quad and $x \notin Q$, then $x$ is collinear with a unique point $\pi_{Q}(x) \in$ $Q$ and we denote by $\mathcal{R}_{Q}(x)$ the unique point of $x \pi_{Q}(x)$ distinct from $x$ and $\pi_{Q}(x)$. If $x \in Q$, then we define $\pi_{Q}(x)=\mathcal{R}_{Q}(x):=x$. The map $x \mapsto \mathcal{R}_{Q}(x)$ defines an automorphism of $Q(5,2) \otimes Q(5,2)$. If $Q_{1}$ and $Q_{2}$ are two disjoint $Q(5,2)$-quads, then the map $Q_{1} \rightarrow Q_{2} ; x \mapsto \pi_{Q_{2}}(x)$ defines an isomorphism between $Q_{1}$ and $Q_{2}$.

(3) There exist two partitions $T_{1}$ and $T_{2}$ of the point set of $Q(5,2) \otimes Q(5,2)$ into $Q(5,2)$-quads.

(4) Every element of $T_{1}$ intersects every element of $T_{2}$ in a line. As a consequence, $S^{\otimes}:=\left\{Q_{1} \cap Q_{2}: Q_{1} \in T_{1}\right.$ and $\left.Q_{2} \in T_{2}\right\}$ is a spread of $Q(5,2) \otimes Q(5,2)$.

(5) For every $Q \in T_{i}, i \in\{1,2\}$, the set $\left\{Q \cap R: R \in T_{3-i}\right\}$ is a spread of symmetry of $Q$.

(6) Every line $L$ of $Q(5,2) \otimes Q(5,2)$ not belonging to $S^{\otimes}$ is contained in a unique quad of $T_{1} \cup T_{2}$.

Now, let $Q$ and $\bar{Q}$ be two disjoint $Q(5,2)$-quads belonging to $T_{1}$ and put $\overline{\bar{Q}}:=$ $\mathcal{R}_{Q}(\bar{Q})=\mathcal{R}_{\bar{Q}}(Q)$. For every point $x$ of $Q$, put $\bar{x}:=\pi_{\bar{Q}}(x)$ and $\overline{\bar{x}}:=\pi_{\bar{Q}}(x)$.

Put $S=\left\{Q \cap Q_{2}: Q_{2} \in T_{2}\right\}$. Then $S$ is a spread of symmetry of $Q$. For every $L \in S$, let $R_{L}$ denote the unique element of $T_{2}$ containing $L$. Let $L^{*}$ denote a specific 
line of $S$ and put $R^{*}=R_{L^{*}}$. For every $L \in S, R_{L} \cap(Q \cup \bar{Q} \cup \overline{\bar{Q}})$ is a $(3 \times 3)$ subgrid $\sigma_{L}$ of $R_{L}$. This $(3 \times 3)$-subgrid $\sigma_{L}$ is contained in precisely three $W(2)$ subquadrangles of $R_{L}$. We denote by $W^{0}, W^{1}, W^{2}$ the three $W(2)$-subquadrangles of $R^{*}$ containing $R^{*} \cap(Q \cup \bar{Q} \cup \overline{\bar{Q}})$. For every $L \in S$ and $i \in \mathbb{Z}_{3}$, put $W_{L}^{i}:=\pi_{R_{L}}\left(W^{i}\right)$.

For every $i \in \mathbb{Z}_{3}$, for every $L \in S$ and for all $x, y \in L$ with $x \neq y$, we denote by $(x, y, i)$ the unique point $\mu$ of $R_{L} \backslash(Q \cup \bar{Q} \cup \overline{\bar{Q}})$ such that $\pi_{Q}(\mu)=x, \pi_{\bar{Q}}(\mu)=\bar{y}$ and $\mu \in W_{L}^{i}$. The point $(x, y, i)$ is the unique point of $W_{L}^{i}$ collinear with $x$ and $\bar{y}$, but not contained in $\sigma_{L}$.

Lemma 3.1 Every point of $Q(5,2) \otimes Q(5,2)$ not contained in $Q \cup \bar{Q} \cup \overline{\bar{Q}}$ has received a unique label.

Proof Let $\mu$ be a point of $Q(5,2) \otimes Q(5,2)$ not contained in $Q \cup \bar{Q} \cup \overline{\bar{Q}}$, let $R$ denote the unique element of $T_{2}$ containing $\mu$ and put $L:=R \cap Q$. Then $R=R_{L}$. There exists a unique $W(2)$-subquadrangle of $R$ containing $\mu$ and $\sigma_{L}$. Let $i \in \mathbb{Z}_{3}$ such that $\mu \in W_{L}^{i}$. Let $x$ and $y$ be the points of $L$ such that $x=\pi_{Q}(\mu)$ and $\bar{y}=\pi \bar{Q}_{Q}(\mu)$. If $x=y$, then $\{x, \bar{y}, \mu\}$ is a set of mutually collinear points, implying that $\mu=\overline{\bar{x}}$, contradicting $\mu \notin \overline{\bar{Q}}$. Hence $x \neq y$ and the point $\mu$ has label $(x, y, i)$. It is also clear that $\mu$ cannot be labeled in different ways.

We will now define a map $\theta: S \times S \rightarrow \mathbb{Z}_{3}$. For each ordered pair $\left(L_{1}, L_{2}\right)$ of lines of $S$, the map $R^{*} \rightarrow R^{*} ; x \mapsto \pi_{R^{*}} \circ \pi_{R_{L_{2}}} \circ \pi_{R_{L_{1}}}(x)$ determines an automorphism of $R^{*}$ fixing each line of the spread $\left\{R^{*} \cap Q_{1}: Q_{1} \in T_{1}\right\}$ of $R^{*}$. By [2, Theorem 4.1], such an automorphism either is trivial or acts on any line of the form $R^{*} \cap Q_{1}, Q_{1} \in$ $T_{1}$, as a cycle. Since every line $R^{*} \cap Q_{1}, Q_{1} \in T_{1} \backslash\{Q, \bar{Q}, \overline{\bar{Q}}\}$, intersects each $W(2)$ subquadrangle $W^{i}, i \in \mathbb{Z}_{3}$, in a unique point, the map $R^{*} \rightarrow R^{*} ; x \mapsto \pi_{R^{*}} \circ \pi_{R_{L_{2}}}$ 。 $\pi_{R_{L_{1}}}(x)$ is either trivial or permutes the elements of $\left\{W^{0}, W^{1}, W^{2}\right\}$ in one of the following ways:

$$
W^{0} \rightarrow W^{1} \rightarrow W^{2} \rightarrow W^{0}, \quad W^{0} \rightarrow W^{2} \rightarrow W^{1} \rightarrow W^{0} .
$$

Hence, there exists a unique $\theta\left(L_{1}, L_{2}\right) \in \mathbb{Z}_{3}$ such that

$$
\pi_{R^{*}} \circ \pi_{R_{L_{2}}} \circ \pi_{R_{L_{1}}}\left(W^{i}\right)=W^{i+\theta\left(L_{1}, L_{2}\right)}
$$

for every $i \in \mathbb{Z}_{3}$.

\section{Lemma 3.2 The following holds:}

(i) For every $L \in S, \theta(L, L)=0$.

(ii) For any two lines $L_{1}$ and $L_{2}$ of $S, \theta\left(L_{2}, L_{1}\right)=-\theta\left(L_{1}, L_{2}\right)$.

(iii) If $L_{1}, L_{2}, L_{3}$ are three lines of $S$ which are contained in a grid, then $\theta\left(L_{1}, L_{2}\right)+$ $\theta\left(L_{2}, L_{3}\right)=\theta\left(L_{1}, L_{3}\right)$.

(iv) If $L_{1}, L_{2}, L_{3}$ are three lines of $S$ which are not contained in a grid, then $\theta\left(L_{1}, L_{2}\right)+\theta\left(L_{2}, L_{3}\right) \neq \theta\left(L_{1}, L_{3}\right)$. 
Proof

(i) For every $i \in \mathbb{Z}_{3}$, we have $\pi_{R^{*}} \circ \pi_{R_{L}} \circ \pi_{R_{L}}\left(W^{i}\right)=\pi_{R^{*}} \circ \pi_{R_{L}}\left(W^{i}\right)=W^{i}$. Hence, $\theta(L, L)=0$.

(ii) If $\pi_{R^{*}} \circ \pi_{R_{L_{2}}} \circ \pi_{R_{L_{1}}}\left(W^{i}\right)=W^{i+\theta\left(L_{1}, L_{2}\right)}$ for every $i \in \mathbb{Z}_{3}$, then $W^{i}=\pi_{R^{*}} \circ$ $\pi_{R_{L_{1}}} \circ \pi_{R_{L_{2}}}\left(W^{i+\theta\left(L_{1}, L_{2}\right)}\right)$ for every $i \in \mathbb{Z}_{3}$. It follows that $\theta\left(L_{2}, L_{1}\right)=$ $-\theta\left(L_{1}, L_{2}\right)$.

(iii) Let $L_{1}, L_{2}, L_{3}$ be three lines of $S$ which are contained in a grid. Then $\pi_{R^{*}} \circ$ $\pi_{R_{L_{3}}} \circ \pi_{R_{L_{1}}}\left(W^{i}\right)=\pi_{R^{*}} \circ \pi_{R_{L_{3}}} \circ \pi_{R_{L_{2}}} \circ \pi_{R_{L_{1}}}\left(W^{i}\right)=\pi_{R^{*}} \circ \pi_{R_{L_{3}}} \circ \pi_{R_{L_{2}}} \circ \pi_{R^{*}} \circ$ $\pi_{R_{L_{2}}} \circ \pi_{R_{L_{1}}}\left(W^{i}\right)=\pi_{R^{*}} \circ \pi_{R_{L_{3}}} \circ \pi_{R_{L_{2}}}\left(W^{i+\theta\left(L_{1}, L_{2}\right)}\right)=W^{i+\theta\left(L_{1}, L_{2}\right)+\theta\left(L_{2}, L_{3}\right)}$. Hence, $\theta\left(L_{1}, L_{3}\right)=\theta\left(L_{1}, L_{2}\right)+\theta\left(L_{2}, L_{3}\right)$.

(iv) Let $L_{1}, L_{2}, L_{3}$ be three lines of $S$ which are not contained in a grid. Suppose that $\theta\left(L_{1}, L_{3}\right)=\theta\left(L_{1}, L_{2}\right)+\theta\left(L_{2}, L_{3}\right)$. Then for every $y \in R^{*}, \pi_{R^{*}} \circ \pi_{R_{L_{3}}} \circ$ $\pi_{R_{L_{1}}}(y)=\left(\pi_{R^{*}} \circ \pi_{R_{L_{3}}} \circ \pi_{R_{L_{2}}}\right) \circ\left(\pi_{R^{*}} \circ \pi_{R_{L_{2}}} \circ \pi_{R_{L_{1}}}\right)(y)$, that is, $\pi_{R^{*}} \circ \pi_{R_{L_{3}}} \circ$ $\pi_{R_{L_{1}}}(y)=\pi_{R^{*}} \circ \pi_{R_{L_{3}}} \circ \pi_{R_{L_{2}}} \circ \pi_{R_{L_{1}}}(y)$. Hence, the map $R_{L_{3}} \rightarrow R_{L_{3}}$ defined by $x \mapsto \pi_{R_{L_{3}}} \circ \pi_{R_{L_{2}}} \circ \pi_{R_{L_{1}}}(x)$ is the identity map on $R_{L_{3}}$. This implies that the points $x, \pi_{R_{L_{1}}}(x), \pi_{R_{L_{2}}}(x)$ are mutually collinear for every $x \in R_{L_{3}}$, that is, $\left\{x, \pi_{R_{L_{1}}}(x), \pi_{R_{L_{2}}}(x)\right\}$ is a line for every $x \in R_{L_{3}}$. This contradicts the fact that $L_{1}, L_{2}, L_{3}$ are not contained in a grid. Hence, $\theta\left(L_{1}, L_{3}\right) \neq \theta\left(L_{1}, L_{2}\right)+$ $\theta\left(L_{2}, L_{3}\right)$.

Proposition 3.3 $Q(5,2) \otimes Q(5,2) \cong \mathcal{S}_{\theta}$, where $\theta$ is as defined above.

Proof We must show that the set of lines of $Q(5,2) \otimes Q(5,2)$ are in bijective correspondence with the sets of Type $(L 1),(L 2), \ldots,(L 9)$ defined in Sect. 2. Obviously:

- The set of lines of $Q(5,2) \otimes Q(5,2)$ contained in $Q$ correspond to the sets of Type (L1).

- The set of lines of $Q(5,2) \otimes Q(5,2)$ contained in $\bar{Q}$ correspond to the sets of Type (L2).

- The set of lines of $Q(5,2) \otimes Q(5,2)$ contained in $\overline{\bar{Q}}$ correspond to the sets of Type (L3).

- The set of lines of $Q(5,2) \otimes Q(5,2)$ meeting $Q, \bar{Q}$ and $\overline{\bar{Q}}$ correspond to the sets of Type (L4).

Consider a line $M$ of $R_{L}$ which is not contained in $\sigma_{L}$ and which intersects $\sigma_{L}$ in a point $a \in L$ of $Q$. Put $L=\{a, b, c\}$. There exists a unique $W(2)$-subquadrangle $W_{L}^{i}$ containing $\sigma_{L}$ and $M$. One readily sees that the points of $M$ have labels $a,(a, b, i)$ and $(a, c, i)$. So, $M$ corresponds to a set of Type (L5). Conversely, every set of Type (L5) corresponds to a (necessarily unique) line of $Q(5,2) \otimes Q(5,2)$.

Next, consider a line $M$ of $R_{L}$ which is not contained in $\sigma_{L}$ and which intersects $\sigma_{L}$ in a point $\bar{a}$ of $\bar{Q}$. Put $L=\{a, b, c\}$. Then, there exists a unique $W(2)$ subquadrangle $W_{L}^{i}$ containing $\sigma_{L}$ and $M$. One readily sees that the points of $M$ have labels $\bar{a},(b, a, i)$ and $(c, a, i)$. So, $M$ corresponds to a set of Type (L6). Conversely, every set of Type (L6) corresponds to a (necessarily unique) line of $Q(5,2) \otimes Q(5,2)$. 
Now, consider a line $M$ of $R_{L}$ which is not contained in $\sigma_{L}$ and which intersects $\sigma_{L}$ in a point $\overline{\bar{a}}$ of $\overline{\bar{Q}}$. Put $L=\{a, b, c\}$. Then, there exists a unique $W(2)$-subquadrangle $W_{L}^{i}$ containing $\sigma_{L}$ and $M$. One readily sees that the points of $M$ have labels $\overline{\bar{a}}$, $(b, c, i)$ and $(c, b, i)$. So, $M$ corresponds to a set of Type ( $L 7)$. Conversely, every set of Type ( $L 7)$ corresponds to a (necessarily unique) line of $Q(5,2) \otimes Q(5,2)$.

Consider next a line $M$ of $R_{L}$ which is disjoint from $\sigma_{L}$. Then $M$ intersects each $W_{L}^{i}, i \in \mathbb{Z}_{3}$, in a unique point. Put $L=\{a, b, c\}$. The labels of the points of $M$ are $\left(u, u^{\prime}, i\right),\left(v, v^{\prime}, j\right),\left(w, w^{\prime}, k\right)$, where $\{i, j, k\}=\{0,1,2\},\{u, v, w\}=$ $\pi_{Q}(M)=\{a, b, c\},\left\{u^{\prime}, v^{\prime}, w^{\prime}\right\}=\pi_{Q} \circ \pi_{\bar{Q}}(M)=\{a, b, c\}, u \neq u^{\prime}, v \neq v^{\prime}, w \neq w^{\prime}$. It readily follows that $\left\{\left(u, u^{\prime}, i\right),\left(v, v^{\prime}, j\right),\left(w, w^{\prime}, k\right)\right\}$ is a set of Type (L8). Conversely, one can readily verify that every set of Type $(L 8)$ corresponds to a line of $Q(5,2) \otimes Q(5,2)$.

Finally, let $M$ be a line of $Q(5,2) \otimes Q(5,2)$ not belonging to $S^{\otimes}$ and contained in a quad of $T_{1} \backslash\{Q, \bar{Q}, \overline{\bar{Q}}\}$. With $M$, there corresponds a set of the form $\{(a, u, i),(b, v, j),(c, w, k)\}$. We have that $\{a, b, c\}=\pi_{Q}(M)$ is a line of $Q$ not belonging to $S$. Similarly, $\{u, v, w\}=\pi_{Q} \circ \pi_{\bar{Q}}(M)$ is a line of $Q$ not belonging to $S$. Moreover, we have that $a u, b v, c w \in S$ and $j=i+\theta(a u, b v), k=i+\theta(a u, c w)$ by the definition of the map $\theta$. So, $M$ corresponds to a set of Type ( $L 9)$. Conversely, we show that every set $\{(a, u, i),(b, v, j),(c, w, k)\}$ of Type (L9) corresponds to a line of $Q(5,2) \otimes Q(5,2)$ not belonging to $S^{\otimes}$ and contained in a quad of $T_{1} \backslash\{Q, \bar{Q}, \overline{\bar{Q}}\}$. Let $x$ denote the point of $Q(5,2) \otimes Q(5,2)$ corresponding to $(a, u, i)$, let $Q_{1}$ denote the unique element of $T_{1}$ containing $x$ and let $M=\pi_{Q_{1}}(\{a, b, c\})$. Then $M$ corresponds to a set of the form $\{(a, u, i),(b, *, *),(c, *, *)\}$. Since $v, w, j, k$ are uniquely determined by $a, u, i, b, c$, this set is equal to $\{(a, u, i),(b, v, j),(c, w, k)\}$.

By the above discussion, we indeed know that $Q(5,2) \otimes Q(5,2) \cong \mathcal{S}_{\theta}$.

\section{Definitions.}

(1) An admissible triple is a triple $\Sigma=(\mathcal{L}, G, \Delta)$, where:

- $G$ is a nontrivial additive group whose order $s+1$ is finite.

- $\mathcal{L}$ is a linear space, different from a point, in which each line is incident with exactly $s+1$ points. We denote the point set of $\mathcal{L}$ by $P$.

- $\Delta$ is a map from $P \times P$ to $G$ such that the following holds for any three points $x, y$ and $z$ of $\mathcal{L}: x, y$ and $z$ are collinear $\Leftrightarrow \Delta(x, y)+\Delta(y, z)=\Delta(x, z)$.

(2) Suppose $\Sigma_{1}=\left(\mathcal{L}_{1}, G_{1}, \Delta_{1}\right)$ and $\Sigma_{2}=\left(\mathcal{L}_{2}, G_{2}, \Delta_{2}\right)$ are two admissible triples, where $\mathcal{L}_{1}$ and $\mathcal{L}_{2}$ are not lines. Then $\Sigma_{1}$ and $\Sigma_{2}$ are called equivalent if there exist an isomorphism $\alpha$ from $\mathcal{L}_{1}$ to $\mathcal{L}_{2}$, an isomorphism $\beta$ from $G_{1}$ to $G_{2}$ and a map $f$ from the point set of $\mathcal{L}_{1}$ to $G_{1}$ satisfying $\Delta_{2}(\alpha(x), \alpha(y))=(f(x)+$ $\left.\Delta_{1}(x, y)-f(y)\right)^{\beta}$ for all points $x$ and $y$ of $\mathcal{L}_{1}$.

Let $\mathcal{L}_{S}$ denote the linear space whose points are the elements of $S$ and whose lines are the unordered triples of lines of $S$ which are contained in a grid, with incidence being containment. Then $\mathcal{L}_{S}$ is isomorphic to the affine plane $\operatorname{AG}(2,3)$ of order three. By Lemma 3.2, we know that $\left(\mathcal{L}_{S}, \mathbb{Z}_{3}, \theta\right)$ is an admissible triple. 
Proposition 3.4 Let $\theta_{1}$ and $\theta_{2}$ be two maps from $S \times S$ to $\mathbb{Z}_{3}$ such that $\Sigma_{1}=$ $\left(\mathcal{L}_{S}, \mathbb{Z}_{3}, \theta_{1}\right)$ and $\Sigma_{2}=\left(\mathcal{L}_{S}, \mathbb{Z}_{3}, \theta_{2}\right)$ are admissible triples. If $\Sigma_{1}$ and $\Sigma_{2}$ are equivalent, then $\mathcal{S}_{\theta_{1}} \cong \mathcal{S}_{\theta_{2}}$.

Proof Since $\Sigma_{1}$ and $\Sigma_{2}$ are equivalent, there exists an automorphism $\alpha$ of $\mathcal{L}_{S}$, an automorphism $\beta$ of $\mathbb{Z}_{3}$ and a map $f$ from $S$ to $\mathbb{Z}_{3}$ satisfying $\theta_{2}(\alpha(x), \alpha(y))=$ $\left(f(x)+\theta_{1}(x, y)-f(y)\right)^{\beta}$ for all points $x$ and $y$ of $\mathcal{L}_{S}$. There exists an automorphism $\phi$ of $Q$ such that $\alpha(L)=\phi(L)$ for every line $L$ of $S$; see, e.g., [3, Sect. 3,

Example 1]. One readily verifies that the map $x \mapsto x^{\phi} ; \bar{x} \mapsto \overline{x^{\phi}} ; \overline{\bar{x}} \mapsto \overline{\overline{x^{\phi}}} ;(a, b, i) \mapsto$ $\left(a^{\phi}, b^{\phi},(i-f(a b))^{\beta}\right)$ defines an isomorphism between $\mathcal{S}_{\theta_{1}}$ and $\mathcal{S}_{\theta_{2}}$.

It is known that the affine plane $\operatorname{AG}(2,3)$ admits, up to equivalence, a unique admissible triple. (This follows, for instance, from [4, Theorem 2.1] and the fact that there exists a unique generalized quadrangle of order $(2,4)$, namely $Q(5,2)$, and a unique spread of symmetry in $Q(5,2)$.) If we coordinatize $\operatorname{AG}(2,3)$ in the standard way, then an admissible triple can be obtained by putting $\Delta\left[\left(x_{1}, y_{1}\right),\left(x_{2}, y_{2}\right)\right]:=$ $x_{1} y_{2}-x_{2} y_{1} \in \mathbb{Z}_{3}$.

\section{A non-abelian representation of the near hexagon $Q(5,2) \times \mathbb{L}_{3}$}

The slim dense near hexagon $Q(5,2) \times \mathbb{L}_{3}$ is obtained by taking three isomorphic copies of $Q(5,2)$ and joining the corresponding points to form lines of size 3 . In this section, we prove that there exists a non-abelian representation of $Q(5,2) \times \mathbb{L}_{3}$.

Let $Q$ and $B$, respectively, be the point and line set of $Q(5,2)$. Set $\bar{Q}=$ $\{\bar{x}: x \in Q\}, \overline{\bar{Q}}=\{\overline{\bar{x}}: x \in Q\}, \bar{B}=\{\{\bar{x}, \bar{y}, \bar{z}\}:\{x, y, z\} \in B\}$ and $\overline{\bar{B}}=\{\{\overline{\bar{x}}, \overline{\bar{y}}, \overline{\bar{z}}\}$ : $\{x, y, z\} \in B\}$. Then $(\bar{Q}, \bar{B})$ and $(\overline{\bar{Q}}, \overline{\bar{B}})$ are isomorphic to $Q(5,2)$. The near hexagon $Q(5,2) \times \mathbb{L}_{3}$ is isomorphic to the geometry whose point set $P$ is $Q \cup \bar{Q} \cup \overline{\bar{Q}}$ and whose line set $L$ is $B \cup \bar{B} \cup \overline{\bar{B}} \cup\{\{x, \bar{x}, \overline{\bar{x}}\}: x \in Q\}$.

It is known that if $Q(5,2) \times \mathbb{L}_{3}$ admits a non-abelian representation, then the representation group must be the extra-special 2-group $2_{+}^{1+12}$ [13, Theorem 1.6, p. 199]. Let $R=2_{+}^{1+12}$ with $R^{\prime}=\{1, \lambda\}$. Set $V=R / R^{\prime}$. Consider $V$ as a vector space over $\mathbb{F}_{2}$. The map $f: V \times V \rightarrow \mathbb{F}_{2}$ defined by

$$
f\left(x R^{\prime}, y R^{\prime}\right)= \begin{cases}0 & \text { if }[x, y]=1, \\ 1 & \text { if }[x, y]=\lambda\end{cases}
$$

for $x, y \in R$, is a non-degenerate symplectic bilinear form on $V$ [5, Theorem 20.4, p. 78]. Write $V$ as an orthogonal direct sum of six hyperbolic planes $K_{i}(1 \leq i \leq 6)$ in $V$ and let $H_{i}$ be the inverse image of $K_{i}$ in $R$ (under the canonical homomorphism $R \rightarrow R / R^{\prime}$ ). Then each $H_{i}$ is generated by two involutions $x_{i}$ and $y_{i}$ such that $\left[x_{i}, y_{i}\right]=\lambda$. Let $M=\left\langle x_{i}: 1 \leq i \leq 6\right\rangle$ and $\bar{M}=\left\langle y_{i}: 1 \leq i \leq 6\right\rangle$. Then $M$ and $\bar{M}$ are elementary abelian 2-subgroups of $R$ each of order $2^{6}$. Further, $M, \bar{M}$ and $Z(R)$ pairwise intersect trivially and $R=M \bar{M} Z(R)$. Also, $C_{M}(\bar{M})$ and $C_{\bar{M}}(M)$ are trivial. 
We regard the points and lines of $Q$ as the points and lines of a nonsingular elliptic quadric of the projective space $\mathrm{PG}(M)$, where $M$ is regarded as a 6dimensional vector space over $\mathbb{F}_{2}$. Let $(M, \tau)$ be the natural abelian representation of $(Q, B)$ associated with this embedding of $Q$ in $\operatorname{PG}(M)$. For every point $x$ of $Q$, put $m_{x}=\tau(x)$. There exists a unique non-degenerate symplectic bilinear form $g$ on $M$ such that $m_{x}^{\perp}=\left\langle m_{y}: y \in x^{\perp}\right\rangle$ for every point $x$ of $Q$; see, e.g., [7, Sect. 22.3]. Here, the following notational convention has been used: for every $m \in M, m^{\perp}$ denotes the set of all $m^{\prime} \in M$ for which $g\left(m, m^{\prime}\right)=0$.

Now, let $m$ be an arbitrary element of $M$. If $m=1$, then we define $\bar{m}:=1$. Suppose now that $m \neq 1$. Then $m^{\perp}$ is maximal in $M$, that is, of index 2 in $M$. So, the centralizer of $m^{\perp}$ in $\bar{M}$ is a subgroup $\langle\bar{m}\rangle$ of order 2. Since $m^{\perp}$ is maximal in $M$, $\left\langle m^{\perp}, m^{\prime}\right\rangle=M$ for every $m^{\prime} \in M \backslash m^{\perp}$. The triviality of $C_{\bar{M}}(M)$ then implies that $\left[\bar{m}, m^{\prime}\right]=\lambda$ for every $m^{\prime} \in M \backslash m^{\perp}$.

We prove that the map $M \rightarrow \bar{M} ; m \mapsto \bar{m}$ is an isomorphism. This map is easily seen to be bijective. (Notice that $C_{M}(\bar{m})=m^{\perp}$.) So, it suffices to prove that $\overline{m_{1} m_{2}}=$ $\overline{m_{1}} \overline{m_{2}}$ for all $m_{1}, m_{2} \in M$. Clearly, this holds if $1 \in\left\{m_{1}, m_{2}\right\}$ or $m_{1}=m_{2}$. So, we may suppose that $m_{1} \neq 1 \neq m_{2} \neq m_{1}$. The set $\left\{m_{1}, m_{2}, m_{1} m_{2}\right\}$ corresponds to a line of $\mathrm{PG}(M)$. So, for every $m \in\left(m_{1} m_{2}\right)^{\perp},\left(\left[\overline{m_{1}}, m\right],\left[\overline{m_{2}}, m\right]\right)$ is equal to either $(1,1)$ or $(\lambda, \lambda)$. Then

$$
\left[\overline{m_{1}} \overline{m_{2}}, m\right]=\left[\overline{m_{1}}, m\right]\left[\overline{m_{2}}, m\right]=1 .
$$

The first equality holds since $R$ has nilpotency class 2 . Thus $\overline{m_{1}} \overline{m_{2}} \in C_{\bar{M}}\left(\left(m_{1} m_{2}\right)^{\perp}\right)$ $=\left\langle\overline{m_{1} m_{2}}\right\rangle$. Since $\overline{m_{1}} \overline{m_{2}} \neq 1$, we have $\overline{m_{1}} \overline{m_{2}}=\overline{m_{1} m_{2}}$.

We conclude that if we define $\bar{\tau}: \bar{Q} \rightarrow \bar{M} ; \bar{x} \mapsto \overline{m_{x}}$ for every $x \in Q$, then $(\bar{M}, \bar{\tau})$ is a faithful abelian representation of $(\bar{Q}, \bar{B})$.

Now, let $m$ be an arbitrary element of $M$. If $m=1$, then we define $\overline{\bar{m}}:=1$. If $m=m_{x}$ for some $x \in Q$, then we define $\overline{\bar{m}}:=m \bar{m}$. If $m \neq 1$ and $m \neq m_{x}, \forall x \in Q$, then we define $\overline{\bar{m}}:=m \bar{m} \lambda$. Since $m^{2}=\bar{m}^{2}=\lambda^{2}=[m, \bar{m}]=1, \overline{\bar{m}}$ is an involution. We prove that the map $m \mapsto \overline{\bar{m}}$ defines an isomorphism between $M$ and an elementary abelian 2-group $\overline{\bar{M}}$ of order $2^{6}$. Since $R=M \bar{M} Z(R)$, this map is injective and hence it suffices to prove that $\overline{\overline{m_{1} m_{2}}}=\overline{\overline{m_{1}}} \overline{\overline{m_{2}}}$ for all $m_{1}, m_{2} \in M$. Obviously, this holds if $1 \in\left\{m_{1}, m_{2}\right\}$ or $m_{1}=m_{2}$. So, we may suppose that $m_{1} \neq 1 \neq m_{2} \neq m_{1}$. The set $\left\{m_{1}, m_{2}, m_{1} m_{2}\right\}$ corresponds to a line of $\mathrm{PG}(M)$. Suppose $3-N$ elements of $\left\{m_{1}, m_{2}, m_{1} m_{2}\right\}$ correspond to points of $Q$. Then $m_{1} \in m_{2}^{\perp}$ if and only if $N$ is even. ${ }^{1}$ So, $\left[\overline{m_{1}}, m_{2}\right]=\lambda^{N}$. If $N^{\prime}$ is the number of elements of $\left\{m_{1}, m_{2}\right\}$ corresponding to points of $Q$, then $2-N^{\prime}-N \in\{-1,0\}$ and $2-N^{\prime}-N=0$ if and only if $m_{1} m_{2}$ corresponds to a point of $Q$. Hence, $\overline{\overline{m_{1}}} \overline{\overline{m_{2}}}=m_{1} \overline{m_{1}} m_{2} \overline{m_{2}} \lambda^{2-N^{\prime}}=$ $m_{1} m_{2} \overline{m_{1}} \overline{m_{2}} \lambda^{2-N^{\prime}-N}=m_{1} m_{2} \overline{m_{1} m_{2}} \lambda^{2-N^{\prime}-N}=\overline{\overline{m_{1} m_{2}}}$.

So, if we define $\overline{\bar{\tau}}: \overline{\bar{Q}} \rightarrow \overline{\bar{M}}$ by putting $\overline{\bar{\tau}}(\overline{\bar{x}}):=\overline{\overline{m_{x}}}=m_{x} \overline{m_{x}}$ for all $x \in Q$, then $(\overline{\bar{M}}, \overline{\bar{\tau}})$ is a faithful abelian representation of $(\overline{\bar{Q}}, \overline{\bar{B}})$.

\footnotetext{
${ }^{1}$ Perhaps the case $N=3$ needs more explanation. Suppose $N=3$ and $m_{1} \in m_{2}^{\perp}$. Then the hyperplane $\pi$ of $\mathrm{PG}(M)$ corresponding to $m \frac{\perp}{2}$ intersects $Q$ in a nonsingular parabolic quadric $Q(4,2)$ of $\pi$. Since the point of $\mathrm{PG}(M)$ corresponding to $m_{2}$ is the kernel of $Q(4,2)$, the line of $\mathrm{PG}(M)$ corresponding to $\left\{m_{1}, m_{2}, m_{1} m_{2}\right\} \subset \pi$ must meet $Q(4,2)$, in contradiction with $N=3$.
} 
Now, define a map $\psi: P \rightarrow R$ which coincides with $\tau$ on $Q, \bar{\tau}$ on $\bar{Q}$ and $\overline{\bar{\tau}}$ on $\overline{\bar{Q}}$. Since $R=\langle M, \bar{M}\rangle, R=\langle\psi(P)\rangle$. By construction, $(R, \psi)$ also satisfies Property (R2) in the definition of representation. Hence, $(R, \psi)$ is a non-abelian representation of $Q(5,2) \times \mathbb{L}_{3}$.

\section{A non-abelian representation of the near hexagon $Q(5,2) \otimes Q(5,2)$}

In this section, we prove that the slim dense near hexagon $Q(5,2) \otimes Q(5,2)$ has a non-abelian representation. By Proposition 3.3, this is equivalent with showing that the partial linear space $\mathcal{S}_{\theta}$ has a non-abelian representation, where $\theta$ is as defined in Sect. 3 .

We continue with the notation introduced in Sect. 3. Let $M^{*}$ be a line of $S^{\otimes}$ contained in $R^{*}$ but distinct from $R^{*} \cap Q, R^{*} \cap \bar{Q}$ and $R^{*} \cap \overline{\bar{Q}}$. Then $M^{*}$ intersects each $W^{i}, i \in\{1,2,3\}$, in a unique point. For every point $x$ of $L^{*}$, put $\epsilon(x):=i$ if the unique point of $M^{*}$ collinear with $x$ belongs to $W^{i}$. If $y \in Q \backslash L^{*}$, then we define $\epsilon(y):=\epsilon(x)$, where $x$ is the unique point of $L^{*}$ collinear with $y$.

Lemma 5.1 Let $L_{1}$ and $L_{2}$ be two distinct lines in $S$ and let $\alpha_{i} \in L_{i}, i \in\{1,2\}$. Then $\alpha_{1} \sim \alpha_{2}$ if and only if $\epsilon\left(\alpha_{2}\right)-\epsilon\left(\alpha_{1}\right)=\theta\left(L_{1}, L_{2}\right)$.

Proof Let $\alpha_{2}^{\prime}$ be the unique point of $L_{2}$ collinear with $\alpha_{1}$, let $x_{1}$ and $x_{2}$ be the unique points of $L^{*}$ nearest to $\alpha_{1}$ and $\alpha_{2}^{\prime}$, respectively, and let $z_{i}, i \in\{1,2\}$, denote the unique point of $M^{*}$ collinear with $x_{i}$. The automorphism $R^{*} \rightarrow R^{*} ; x \mapsto \pi_{R^{*}} \circ \pi_{R_{L_{2}}} \circ$ $\pi_{R_{L_{1}}}(x)$ of $R^{*}$ maps $x_{1}$ to $x_{2}$ and hence $z_{1}$ to $z_{2}$. This implies that $W^{\epsilon\left(x_{1}\right)+\theta\left(L_{1}, L_{2}\right)}=$ $W^{\epsilon\left(x_{2}\right)}$, i.e., $\theta\left(L_{1}, L_{2}\right)=\epsilon\left(x_{2}\right)-\epsilon\left(x_{1}\right)=\epsilon\left(\alpha_{2}^{\prime}\right)-\epsilon\left(\alpha_{1}\right)$. Hence, $\alpha_{1} \sim \alpha_{2}$ if and only if $\alpha_{2}=\alpha_{2}^{\prime}$, i.e., if and only if $\epsilon\left(\alpha_{2}\right)-\epsilon\left(\alpha_{1}\right)=\theta\left(L_{1}, L_{2}\right)$.

Lemma 5.2 Let $N=2_{-}^{1+6}$ with $N^{\prime}=\{1, \lambda\}$ and let $I_{2}(N)$ be the set of involutions in $N$. Then there exists a map $\delta$ from $Q$ to $I_{2}(N)$ satisfying the following:

(i) $\delta$ is one-one.

(ii) For $x, y \in Q,[\delta(x), \delta(y)]=1$ if and only if $y \in x^{\perp}$.

(iii) If $x, y \in Q$ with $x \sim y$, then

$$
\delta(x * y)= \begin{cases}\delta(x) \delta(y) & \text { if } x y \in S, \\ \delta(x) \delta(y) \lambda & \text { if } x y \notin S .\end{cases}
$$

(iv) The image of $\delta$ generates $N$.

Proof We use a model for the generalized quadrangle $Q \cong Q(5,2)$ which is described in [11, Sect. 6.1, pp. 101-102]. Put $\Omega=\{1,2,3,4,5,6\}$ and $\Omega^{\prime}=$ $\left\{1^{\prime}, 2^{\prime}, 3^{\prime}, 4^{\prime}, 5^{\prime}, 6^{\prime}\right\}$. Let $\mathcal{E}$ be the set of all 2-subsets of $\Omega$ and let $\mathcal{F}$ be the set of all partitions of $\Omega$ in three 2-subsets of $\Omega$. Then the point set of $Q$ can be identified with the set $\mathcal{E} \cup \Omega \cup \Omega^{\prime}$ and the line set of $Q$ can be identified with the set 
$\mathcal{F} \cup\left\{\left\{i,\{i, j\}, j^{\prime}\right\}: 1 \leq i, j \leq 6, i \neq j\right\}$. Now, consider the following nine lines of $Q$ :

$$
\begin{array}{lll}
L_{1}=\{\{1,2\},\{3,4\},\{5,6\}\} ; & L_{2}=\left\{\{1,4\}, 1,4^{\prime}\right\} ; & L_{3}=\left\{\{2,6\}, 2,6^{\prime}\right\} ; \\
L_{4}=\{\{1,6\},\{2,4\},\{3,5\}\} ; & L_{5}=\left\{\{1,5\}, 1^{\prime}, 5\right\} ; & L_{6}=\left\{\{2,3\}, 2^{\prime}, 3\right\} ; \\
L_{7}=\{\{1,3\},\{2,5\},\{4,6\}\} ; & L_{8}=\left\{\{3,6\}, 3^{\prime}, 6\right\} ; & L_{9}=\left\{\{4,5\}, 4,5^{\prime}\right\} .
\end{array}
$$

These 9 lines are mutually disjoint and hence determine a spread $S^{\prime}$ of $Q$. Any two distinct lines $L_{i}$ and $L_{j}$ of $S^{\prime}$ are contained in a unique $(3 \times 3)$-subgrid and the unique line of this subgrid disjoint from $L_{i}$ and $L_{j}$ also belongs to $S^{\prime}$. A spread of $Q(5,2)$ having this property is called regular. Since any regular spread of $Q(5,2)$ is also a spread of symmetry [2, Sect. 7.1], and there exists up to isomorphism a unique spread of symmetry in $Q(5,2)$, we may without loss of generality suppose that $S=S^{\prime}$.

Put $N=\langle a, b\rangle \circ\langle c, d\rangle \circ Q_{8}$, where $a, b, c, d$ are involutions and $\langle a, b\rangle \cong\langle c, d\rangle \cong$ $D_{8}$. So, $[a, b]=[c, d]=\lambda$. Take $Q_{8}=\{1, \lambda, i, j, k, i \lambda, j \lambda, k \lambda\}$, where $i^{2}=j^{2}=$ $k^{2}=\lambda, i j=k, j k=i, k i=j$ and $[i, j]=[j, k]=[k, i]=\lambda$. We define $\delta: Q \rightarrow$ $I_{2}(N)$ as follows:

$$
\begin{aligned}
& \delta(\{1,2\})=a, \quad \delta(\{3,4\})=c, \quad \delta(\{5,6\})=a c, \\
& \delta(\{1,4\})=a b d i, \quad \delta(1)=c d j, \quad \delta\left(4^{\prime}\right)=a b c k \lambda, \\
& \delta(\{2,6\})=a b i \lambda, \quad \delta(2)=a c d k, \quad \delta\left(6^{\prime}\right)=b c d j \lambda, \\
& \delta(\{1,6\})=b, \quad \delta(\{2,4\})=b d, \quad \delta(\{3,5\})=d, \\
& \delta(\{1,5\})=a b c i, \quad \delta\left(1^{\prime}\right)=c d k \lambda, \quad \delta(5)=a b d j, \\
& \delta(\{2,3\})=b c d i \lambda, \quad \delta\left(2^{\prime}\right)=a c d j \lambda, \quad \delta(3)=a b k, \\
& \delta(\{1,3\})=a b c d \lambda, \quad \delta(\{2,5\})=b c \lambda, \quad \delta(\{4,6\})=a d \lambda, \\
& \delta(\{3,6\})=a c d i \lambda, \quad \delta\left(3^{\prime}\right)=a b j \lambda, \quad \delta(6)=b c d k, \\
& \delta(\{4,5\})=c d i, \quad \delta(4)=a b c j, \quad \delta\left(5^{\prime}\right)=a b d k \lambda .
\end{aligned}
$$

Put $W=N / N^{\prime}$. Suppose $\left\{x_{1}, x_{2}, \ldots, x_{6}\right\}$ is a set of 6 points of $Q$ such that the smallest subspace $\left[x_{1}, x_{2}, \ldots, x_{6}\right]$ of $Q$ containing $\left\{x_{1}, x_{2}, \ldots, x_{6}\right\}$ coincides with $Q$. If $\tau$ is an abelian representation of $Q$ in $W$, then by Property (R1) in the definition of representation, $W=\left\langle\tau\left(x_{1}\right), \ldots, \tau\left(x_{6}\right)\right\rangle$ and hence $\left\{\tau\left(x_{1}\right), \ldots, \tau\left(x_{6}\right)\right\}$ is a basis of $W$ (regarded as $\mathbb{F}_{2}$-vector space). Conversely, if $\left\{w_{1}, \ldots, w_{6}\right\}$ is a basis of $W$, then the map $x_{i} \mapsto w_{i}, i \in\{1, \ldots, 6\}$, can be extended to a unique abelian representation $\tau$ of $Q$ in $W$. (Since there exists an abelian representation of $Q$ in $W$, there must exist an abelian representation $\tau$ for which $\tau\left(x_{i}\right)=w_{i}, i \in\{1, \ldots, 6\}$. The uniqueness of $\tau$ follows from the fact that $\tau\left(y_{1} * y_{2}\right)=\tau\left(y_{1}\right) \tau\left(y_{2}\right)$ for any two distinct collinear points $y_{1}$ and $y_{2}$ of $Q$.) Consider now the special case where $x_{1}=\{1,2\}, x_{2}=\{3,4\}$, $x_{3}=\{3,5\}, x_{4}=\{1,6\}, x_{5}=\{4,5\}, x_{6}=1, w_{1}=a N^{\prime}, w_{2}=c N^{\prime}, w_{3}=d N^{\prime}, w_{4}=$ $b N^{\prime}, w_{5}=c d i N^{\prime}$ and $w_{6}=c d j N^{\prime}$. One indeed readily verifies that $\left[x_{1}, \ldots, x_{6}\right]=Q$ and that $\left\{w_{1}, \ldots, w_{6}\right\}$ is a basis of the $\mathbb{F}_{2}$-vector space $W$. Let $\delta^{\prime}$ denote the unique abelian representation of $Q$ in $W$ for which $\delta^{\prime}\left(x_{i}\right)=w_{i}, i \in\{1, \ldots, 6\}$. Then, using 
the fact that $\delta^{\prime}\left(y_{1} * y_{2}\right)=\delta^{\prime}\left(y_{1}\right) \delta^{\prime}\left(y_{2}\right)$ for any two distinct collinear points $y_{1}$ and $y_{2}$ of $Q$, one can verify that $\delta^{\prime}(y)=\delta(y) N^{\prime}$ for every $y \in Q$. This implies that $\delta\left(y_{1} * y_{2}\right)$ is equal to either $\delta\left(y_{1}\right) \delta\left(y_{2}\right)$ or $\delta\left(y_{1}\right) \delta\left(y_{2}\right) \lambda$ for any two distinct collinear points $y_{1}$ and $y_{2}$ of $Q$.

Clearly, the map $\delta: Q \rightarrow I_{2}(N)$ satisfies the properties (i) and (iv) of the lemma. We will now prove that also property (ii) of the lemma is satisfied. So, if $\left\{y_{1}, y_{2}\right\}$ is one of the 351 unordered pairs of distinct points of $Q$, then we need to prove that $\left[\delta\left(y_{1}\right), \delta\left(y_{2}\right)\right]=1$ if and only if $y_{1} \in y_{2}^{\perp}$. Since $Q=\left[x_{1}, \ldots, x_{6}\right]$, it suffices to prove the following three statements: (I) the above claim holds if $\left\{y_{1}, y_{2}\right\} \subseteq\left\{x_{1}, \ldots, x_{6}\right\}$; (II) if $\left[\delta\left(y_{1}\right), \delta\left(y_{2}\right)\right]=1$ for some distinct collinear points $y_{1}$ and $y_{2}$, then also $\left[\delta\left(y_{1}\right), \delta\left(y_{1} * y_{2}\right)\right]=1$; (III) if the above claim holds for unordered pairs $\left\{y_{1}, y_{2}\right\}$ and $\left\{y_{1}, y_{3}\right\}$ of points where $y_{2} \sim y_{3}$ and $y_{1} \notin y_{2} y_{3}$, then it also holds for the unordered pair $\left\{y_{1}, y_{2} * y_{3}\right\}$. Statement (I) is easily verified by considering all 15 pairs $\left\{x_{i}, x_{j}\right\}$ where $i, j \in\{1, \ldots, 6\}$ with $i \neq j$. As to Statement (II), notice that $\left[\delta\left(y_{1}\right), \delta\left(y_{1} * y_{2}\right)\right]$ is equal to either $\left[\delta\left(y_{1}\right), \delta\left(y_{1}\right) \delta\left(y_{2}\right)\right]$ or $\left[\delta\left(y_{1}\right), \delta\left(y_{1}\right) \delta\left(y_{2}\right) \lambda\right]$ which is in any case equal to 1 . We now prove Statement (III). Since $\delta\left(y_{2} * y_{3}\right)$ is equal to either $\delta\left(y_{2}\right) \delta\left(y_{3}\right)$ or $\delta\left(y_{2}\right) \delta\left(y_{3}\right) \lambda$, we have $\left[\delta\left(y_{1}\right), \delta\left(y_{2} * y_{3}\right)\right]=\left[\delta\left(y_{1}\right), \delta\left(y_{2}\right) \delta\left(y_{3}\right)\right]=\left[\delta\left(y_{1}\right), \delta\left(y_{2}\right)\right]\left[\delta\left(y_{1}\right), \delta\left(y_{3}\right)\right]$. If $y_{1}$ is collinear with precisely one of $y_{2}, y_{3}$, then $y_{1}$ is not collinear with $y_{2} * y_{3}$ and $\left[\delta\left(y_{1}\right), \delta\left(y_{2} * y_{3}\right)\right]=\left[\delta\left(y_{1}\right), \delta\left(y_{2}\right)\right]\left[\delta\left(y_{1}\right), \delta\left(y_{3}\right)\right]=1 \cdot \lambda=\lambda$. If $y_{1}$ is collinear with $y_{2} * y_{3}$, then $\left[\delta\left(y_{1}\right), \delta\left(y_{2} * y_{3}\right)\right]=\left[\delta\left(y_{1}\right), \delta\left(y_{2}\right)\right]\left[\delta\left(y_{1}\right), \delta\left(y_{3}\right)\right]=\lambda \cdot \lambda=1$. So, this proves Statement (III) and finishes the proof of property (ii) of the lemma.

Property (iii) of the lemma is verified by considering all 45 lines $L$ of $Q$ and an ordered pair $(x, y)$ of distinct points of $L$. Notice that by property (ii) of the lemma, we only need to consider one ordered pair $(x, y)$ for each line $L$ of $Q$.

It is known that if the near hexagon $Q(5,2) \otimes Q(5,2)$ admits a non-abelian representation, then the representation group must be the extra-special 2-group $2_{-}^{1+18}[13$, Theorem 1.6, p. 199]. We next construct a non-abelian representation of $\mathcal{S}_{\theta} \cong Q(5,2) \otimes Q(5,2)$ in the group $2_{-}^{1+18}$.

Let $R=2_{-}^{1+18}$ with $R^{\prime}=\{1, \lambda\}$. Write $R$ as a central product $R=M \circ N$, where $M=2_{+}^{1+12}$ and $N=2_{-}^{1+6}$. Let $Y=Q \cup \bar{Q} \cup \overline{\bar{Q}}$. Then the subgeometry of $\mathcal{S}_{\theta}$ whose point set is $Y$ together with the lines of types $(L 1)-(L 4)$ is isomorphic to $Q(5,2) \times$ $\mathbb{L}_{3}$. Let $P$ be the point set of $\mathcal{S}_{\theta}$ and let $\delta$ be a map from $Q$ to $I_{2}(N)$ satisfying the conditions of Lemma 5.2. We extend $\delta$ to the set $P \backslash Y$ using the map $\epsilon: Q \rightarrow \mathbb{Z}_{3}$ which we defined in the beginning of this section:

For $L_{1} \in S$, distinct points $a, b \in L_{1}$ and $j \in \mathbb{Z}_{3}$, we define $\delta(a, b, j):=\delta(u)$, where $u$ is the unique point of $L_{1}$ with $\epsilon(u)=j$.

Now, fix a non-abelian representation $(M, \phi)$ of $Y$. Such a representation exists by Sect. 4. Let $\psi$ be the following map from $P$ to $R$ :

- If $q \in Y$, then $\psi(q):=\phi(q)$.

- If $q=(a, b, i) \in P \backslash Y$, then $\psi(q)=\psi(a, b, i):=\phi(b) \phi(\bar{a}) \delta(a, b, i)$.

We prove the following. 
Theorem $5.3(R, \psi)$ is a non-abelian representation of $\mathcal{S}_{\theta}$.

Proof Since the image of $\phi$ generates $M$ and the image of $\delta$ generates $N$, we have $R=\langle\psi(P)\rangle$. For every line $L_{1} \in S$ and distinct $a, b \in L_{1}$, we have $[\phi(a), \phi(\bar{b})]=1$, since $a$ and $\bar{b}$ are at distance two from each other. This implies that $\psi(q)$ is an involution for every $q \in P$. We need to verify Condition $(R 2)$ in the definition of representation. This is true for all lines of types $(L 1)-(L 4)$, since they are also lines of $Y$ and $\psi$ coincides with $\phi$ on $Y$.

Let $\{a,(a, b, i),(a, c, i)\}$ be a line of type (L5). Since $\delta(a, b, i)=\delta(a, c, i)$, we have $\psi(a, b, i) \psi(a, c, i)=\phi(b) \phi(\bar{a}) \phi(c) \phi(\bar{a})=\phi(b) \phi(c)=\phi(a)=\psi(a)$. Similar argument holds for lines of types (L6) and (L7).

Next, consider a line $\{(a, b, i),(b, c, j),(c, a, k)\}$ of type (L8). We have $\psi(a, b, i) \psi(b, c, j)=\phi(b) \phi(\bar{a}) \phi(c) \phi(\bar{b}) \delta(a, b, i) \delta(b, c, j)$. Since $\{i, j, k\}=\mathbb{Z}_{3}$, $\{\delta(a, b, i), \delta(b, c, j), \delta(c, a, k)\}=\{\delta(a), \delta(b), \delta(c)\}$. Since $\{a, b, c\} \in S$, Lemma 5.2(iii) implies that $\delta(a, b, i) \delta(b, c, j)=\delta(c, a, k)$. So, $\psi(a, b, i) \psi(b, c, j)=$ $\phi(b) \phi(c) \phi(\bar{a}) \phi(\bar{b}) \delta(c, a, k)=\phi(a) \phi(\bar{c}) \delta(c, a, k)=\psi(c, a, k)$. Notice that the second equality holds since $\{a, b, c\}$ and $\{\bar{a}, \bar{b}, \bar{c}\}$ are lines of $Y$.

Finally, consider a line $\{(a, u, i),(b, v, j),(c, w, k)\}$ of type (L9). Here the lines $a u, b v, c w$ are in $S, j=i+\theta(a u, b v)$ and $k=i+\theta(a u, c w)$. Let $\delta(a, u, i)=$ $\delta(\alpha), \delta(b, v, j)=\delta(\beta)$ and $\delta(c, w, k)=\delta(\gamma)$, where $\alpha \in a u, \beta \in b v$ and $\gamma \in c w$. So $\epsilon(\alpha)=i, \epsilon(\beta)=j$ and $\epsilon(\gamma)=k$. Since $\epsilon(\beta)-\epsilon(\alpha)=j-i=\theta(a u, b v)$, Lemma 5.1 implies that $\alpha \sim \beta$. Similarly, $\alpha \sim \gamma$. Thus $\{\alpha, \beta, \gamma\}$ is a line of $Q$ not contained in $S$. Then by Lemma 5.2(iii), $\delta(a, u, i) \delta(b, v, j)=\delta(\alpha) \delta(\beta)=\delta(\gamma) \lambda=\delta(c, w, k) \lambda$. So $\psi(a, u, i) \psi(b, v, j)=\phi(u) \phi(\bar{a}) \phi(v) \phi(\bar{b}) \delta(c, w, k) \lambda$. Since $v$ and $\bar{a}$ are at distance three from each other, $[\phi(\bar{a}), \phi(v)]=\lambda$. So $\phi(\bar{a}) \phi(v)=\phi(v) \phi(\bar{a})[\phi(\bar{a})$, $\phi(v)]=\phi(v) \phi(\bar{a}) \lambda$. Then $\psi(a, u, i) \psi(b, v, j)=\phi(u) \phi(v) \phi(\bar{a}) \phi(\bar{b}) \delta(c, w, k)=$ $\phi(w) \phi(\bar{c}) \delta(c, w, k)=\psi(c, w, k)$. The second equality holds since $\{\bar{a}, \bar{b}, \bar{c}\}$ and $\{u, v, w\}$ are lines of $Y$. This completes the proof.

\section{References}

1. Brouwer, A.E., Cohen, A.M., Hall, J.I., Wilbrink, H.A.: Near polygons and Fischer spaces. Geom. Dedic. 49, 349-368 (1994)

2. De Bruyn, B.: Generalized quadrangles with a spread of symmetry. Eur. J. Comb. 20, 759-771 (1999)

3. De Bruyn, B.: On the number of nonisomorphic glued near hexagons. Bull. Belg. Math. Soc. Simon Stevin 7, 493-510 (2000)

4. De Bruyn, B., Thas, K.: Generalized quadrangles with a spread of symmetry and near polygons. Ill. J. Math. 46, 797-818 (2002)

5. Doerk, K., Hawkes, T.: Finite Soluble Groups. de Gruyter Expositions in Mathematics, vol. 4. Walter de Gruyter, Berlin (1992)

6. Gorenstein, D.: Finite Groups. Chelsea, New York (1980)

7. Hirschfeld, J.W.P., Thas, J.A.: General Galois Geometries. Oxford Mathematical Monographs. Oxford Science Publications. Clarendon, New York (1991)

8. Ivanov, A.A.: Non-abelian representations of geometries. In: "Groups and Combinatorics"-In Memory of Michio Suzuki. Adv. Stud. Pure Math., vol. 32, pp. 301-314. Math. Soc. Japan, Tokyo (2001)

9. Ivanov, A.A., Pasechnik, D.V., Shpectorov, S.V.: Non-abelian representations of some sporadic geometries. J. Algebra 181, 523-557 (1996)

10. Patra, K.L., Sahoo, B.K.: A non-abelian representation of the dual polar space $D Q(2 n, 2)$. Innov. Incidence Geom. 9, 177-188 (2009) 
11. Payne, S.E., Thas, J.A.: Finite Generalized Quadrangles, 2nd edn. EMS Series of Lectures in Mathematics. European Mathematical Society (EMS), Zürich (2009)

12. Sahoo, B.K., Sastry, N.S.N.: A characterization of finite symplectic polar spaces of odd prime order. J. Comb. Theory, Ser. A 114, 52-64 (2007)

13. Sahoo, B.K., Sastry, N.S.N.: On the order of a non-abelian representation group of a slim dense near hexagon. J. Algebraic Comb. 29, 195-213 (2009) 